\title{
Multisensory environmental therapy (Snoezelen) for job stress reduction in mental health nurses: a randomized trial
}

\begin{abstract}
This independent measures experimental design study conducted in the UK and USA, assessed the benefits of multisensory environmental therapy (MSET) using a snoezelen room to reduce occupational stress in sixteen mental health nurses. An independent staff member randomly allocated each participant to one of two intervention conditions: either the hospital /university unit lounge (control group $n=8$ ) or MSET (experimental group $\mathrm{n}=8$ ). The intervention was undertaken for four weeks, two sessions per week, for thirty minutes. Measures of pulse rate were obtained both preand post-intervention. In addition, pre-and post-measures were obtained on the StateTrait Anxiety Scale and the Profile of Mood States and, upon conclusion of the study, an intervention satisfaction questionnaire was completed. Sixteen participants were included in the analysis of the primary outcomes. A significant treatment by pre-post interaction was found for pulse rates, the State Anxiety Scale, and on the Confusion Bewilderment sub-scale of the Profile of Mood States scale. In conclusion, nurses reported unanimous satisfaction with MSET and felt their on-the-job performance was enhanced in the areas of holistic caring for patients and problem solving.
\end{abstract}

Keywords: burnout, stress, multisensory, treatment, questionnaire
Volume II Issue I - 2018

Lesley Collier,' Jason Staal, ${ }^{2}$ Peter Homel $^{2}$

'Faculty of Health Sciences, University of Southampton, UK

${ }^{2}$ Mount Sinai Beth Israel Medical Centre, USA

Correspondence: Lesley Collier, Senior Lecturer in Occupational Therapy, Programme \& Professional Lead for Occupational Therapy, Centre for Innovation and Leadership in Health Sciences, Room 2029, Building 45, USA, Email Lesley.Collier@soton.ac.uk

Received: November 17, 2017 | Published: February 09, 2018

\section{Introduction}

Occupational stress is a widely recognized in vocational rehabilitation. In particular, it is a problem for nurses in the health care industry. ${ }^{1,2}$ Factors such as work overload, paperwork and patient documentation, exposure to violence, changing staff levels, job role diffusion, administrative duties, career development issues, coping with patient illness and death, and bringing work problems home contribute to high levels of on-the-job stress and a challenge to holistic nursing. ${ }^{1,3}$ Chronic job stress can lead to negative psychological states described as burnout. Burnout has been described as a syndrome of emotional exhaustion, depersonalization, and reduced personalized accomplishment. ${ }^{4}$ Burnout is correlated with negative health effects; physical illness, exhaustion, increased alcohol or drug use, marital and/or family problems, anxiety and depression. ${ }^{5}$ Burnout, in turn, negatively affects the delivery of services to patients; reduced quality of nursing care, greater frequency of both absenteeism and job turnover. Burnout rates in younger nurses are high which lead many to drop out of the field. ${ }^{6}$ Methods of alleviating nursing occupational stress to reduce burnout have targeted the identification and modification of occupational stressors, developing coping strategies to manage stress, and interventions developed to reduce stress. ${ }^{7-9}$

Relaxation training is a common intervention used to reduce stress and avoid burnout. Researchers in the field of nursing have emphasized the use of relaxation techniques as a means to reduce on-the-job stress. ${ }^{7}$ Relaxation procedures can be divided into two types: approaches that involve progressive relaxation of muscles, and those that use cognitive methods involving imagery, meditation, and biofeedback. ${ }^{9}$ Stress reduction interventions have been demonstrated to be effective; their use however may be limited by prohibiting factors. Techniques such as progressive muscle relaxation and cognitive approaches require prior instruction before nurses can implement them and derive any benefit. Other stress reduction interventions, for example, massage therapy and social support groups; require the presence of other people to which nurses may not have ready access to. In addition to prior training, techniques such as meditation or yoga require changes in daily routine and deliberate effort to maintain the new healthy behavior.

In the present study, the effects of a novel use of MSET, on nurses were explored. Dutch practitioners first developed the multisensory, reinforcement and relaxation environment for use with developmentally disabled children. They coined the term Snoezelen ${ }^{10}$ to refer to it, melding the two Dutch words for seeking out/taking in and relaxation. ${ }^{11}$ The MSET environment consists of a sensory treatment room outfitted with a variety of sensory stimulation equipment designed to target the four senses of vision, hearing, touch, and smell. The intent is to provide individualized gentle rewarding sensory stimulation without the need for higher cognitive processes, such as learning or memory, in a non-threatening environment available to nurses on-the-job. Prior research in many of the MSE or snoezelen studies have found consistent results: reduction of anxiety, improvement in mood, reduction of agitation, a reduction in pulse rates, a physiological indicator of the relaxation response, and was an enjoyable intervention to experience when assessed across a diverse range of populations; patients with dementia, children with developmental disabilities, and adults with chronic pain and psychiatric disorders. ${ }^{12-16}$

The theoretical framework underlying MSET is the operant paradigm of automatic reinforcement ${ }^{17,18}$ and the physiologically based relaxation response. ${ }^{19}$ Automatic reinforcement refers to events in which behavioral responses are sustained by operant processes independent of any mediation by other people. Sensory reinforcement refers to classes of unconditioned sensory stimuli/events that produce observable consequences of responding, reinforcement, in: animals, normal children, developmentally disabled and psychotic children, 
and normal adults..$^{20-22}$ The multi-sensory stimulation is theorized to be effective as reinforcement because it uses classes of automatic stimuli (sensory experiences) that are matched to the preferences of the person. ${ }^{23}$ The experience of positive affect evoked by reward is thought to change the importance of prominent contextual stimuli that in turn directs the course of behavior. ${ }^{24}$

The relaxation response is a positive physiological reaction evoked by a combination of cognitive, affective and environmental factors which have the therapeutic effect of lowering blood pressure and reducing the subjective experience of stress. The relaxation response can be evoked by a variety of relaxation techniques such as breathing, progressive muscle relaxation, meditation, massage therapy, music therapy and imagery. ${ }^{7,25}$ The relaxation response is theorized to occur in MSET due to discriminated responding to the properties of multiple stimuli, which reduces the probability of the participant experiencing negative cognitions and affect, and increases mindfulness. ${ }^{26}$ Relaxation techniques may be conceptualized by negative reinforcement, people learn to relax in order to avoid or escape negative experiences, such as anxiety and stress. The likelihood of acquiring the relaxation response and continued adherence to use of a technique may be greater if the relaxation intervention is based on a model of positive reinforcement, whereby a person experiences the process of relaxing as rewarding. Current relaxation approaches produce calm but do not target enhancement of positive affect as a primary treatment goal. Positive affect itself may produce mitigating effects against stress ${ }^{27}$ and has been demonstrated to increase clinical problem-solving abilities. ${ }^{28}$ Based on prior empirical work on MSESnoezelen, it was hypothesized that there would be benefits to nurses, who may experience high rates on-the-job stress. It was predicted that participants randomized to receive MSET would have a decrease in pulse rates compared to nurses who were provided time to relax in the nurse unit lounge. It was assumed that MSET is more effective in reducing negative effect in nurses compared with time nurses spent in the unit lounge It was surmised that nurses using MSET would show improved mood compared with nurses who are provided access to a lounge. It was predicted that the reward of MSET is satisfying to experience.

\section{Methods}

\section{Participants}

This independent measures experimental design study recruited sixteen female nurses involved in caring for adults with mental health problems and older people with Alzheimer's disease based in an acute care, psychiatric inpatient unit from a large urban hospital based in the USA and Mental Health Services based in the UK. Participants were recruited on a voluntary basis via an information sheet detailing the study and process to register interest. Participants who fulfilled the entry criteria were randomly allocated by an independent member of staff employed within each hospital setting. Entry criteria included employment on a full time basis as a nurse working within a psychiatric/mental health unit for older people. The two participatory groups comprised of nurses ranging in age from 24 to 61 years (mean=42.75), 13 were White, 2 were African-American, and 1 was Asian. The participants attended sessions on their own time, during their lunch breaks or upon finishing their day shift. The nurses were randomly assigned to either of two treatment conditions: the normal unit lounge $(\mathrm{n}=8)$ or MSET $(\mathrm{n}=8)$. Informed written consent and a HIPAA form (Health Insurance Portability and Accountability Act of 1996) were appropriate was obtained from all participants and the study was approved by the Institutional Review Boards and local Ethics Committees.

\section{Measurements}

Instruments for this study included the State Trait Anxiety Inventory (STAI), the Profile of Mood States (POMS), physiologic measurement of pulse, and a satisfaction questionnaire.

Physiological measurement: Nurses measured their own pulses in order to evaluate the relaxation response. The determination was made that the nurse could record their own pulses secondary to the expertise they have in this area. Each nurse was queried as to their ability to read their own pulse and was instructed to come to each session with a wrist watch. The approach was standardized by having each nurse locate their pulse using the radical artery on the wrist and using index and middle fingers together for measurement.

Anxiety: The State-Trait Anxiety Inventory (STAI) was used to measure anxiety. It is a 40 -item self-reports that has been used to measure anxiety in prior job stress studies. Its reliability ranges from .83 to .92 and has been shown to have concurrent validity. ${ }^{29}$ The STAI yields separate scores for state and trait anxiety.

Mood: Mood was assessed with the Profile of Mood States (POMS). The POMS scale consists of 65 -items, which assess six subjective affective states: tension-anxiety, depression, anger, vigor, fatigue and confusion. The POMS has been used in numerous stress and coping studies and shows very good internal consistency with Cronbach's alpha $=.95 .{ }^{30}$ The POMS provides a total mood disturbance in addition to scores for tension-anxiety, depression, confusion, and vigor.

Satisfaction: In order to assess the perceived benefits of the interventions, a 12-item satisfaction questionnaire designed for this study assessed participant satisfaction.

\section{Procedures}

The nurses were randomly assigned by an independent member of staff to either of two treatment conditions: the standard unit lounge (Control $\mathrm{n}=8$ ) or MSET (Experimental $\mathrm{n}=8$ ). The intervention, consisting of either the unit lounge or the MSET treatment room, was undertaken for four weeks, two sessions per week for thirty minutes per session. A research assistant would meet each nurse on the inpatient unit at a set time prior to each session, whereupon the nurse would be asked to record her pulse and fill out the STAI and the POMS. Each nurse was then escorted to their assigned treatment room by the researcher. Nurses who experienced the unit lounge were accompanied to the unit lounge by the researcher who then left once the nurse was in the lounge. The unit lounge is a designated location situated outside the inpatient unit. The unit lounge is comprised of one couch, several chairs, coffee table, and a boom box for music, microwave, refrigerator, window, and lockers. No restrictions were placed on the nurses who attended the unit lounge except for the stipulated requirements of session frequency and length in order to capture the normal effects of the lounge. The treatment administered to the MSET group began with a pre-test to determine the sensory preferences of each nurse, thus individualizing the treatment. ${ }^{23}$ Each nurse in the MSET group partook in sensory preference assessment of sensory stimuli for the senses of vision, audition, smell and touch. ${ }^{23}$ Nurses in the MSET group rated their preferences to selected stimuli of vision, touch, scent and music using a SUDS (subjective units of discomfort) scale (1=least preferred, $10=$ most preferred). Each nurse was asked to bring in their own musical selection if the available music did not suit their taste. The researchers then set up the MSET treatment room based on the nurse's preference assessment initial assessment. Overhead lighting was gradually faded until the room was only lit by light generated from Snoezelen equipment. The music selection 
chosen by the nurse was turned on, the nurse's preferred aroma therapy scent was set up, and the pre-selected tactile stimuli were placed near the nurse who sat on a cushioned chair. The researcher then left the treatment room and closed the door. During the intervention nurses attended the MSET treatment room individually. Upon conclusion of the designated thirty minutes; which was monitored with a digital stop watch, the researcher re-entered the lounge or MSET treatment room and asked the nurse to repeat the assessment process (pulse, STAI, and POMS). Upon conclusion of the last session of either intervention, each nurse was asked to complete the satisfaction questionnaire. No changes were made to trial outcomes after the trial commenced.

\section{Statistical analysis}

A four-way repeated measures ANOVA compared treatment (lounge versus MSET), week (1 to 4), session within week (1 versus 2 ) and measurement (pre versus post) was used to analyze results for pulse rate, STAI, and the POMS. The test for the interaction of treatment $x$ measurement addressed the general hypothesis of whether MSET was more effective in reducing pulse rate and anxiety, while improving the mood of the nurses. In addition, the treatment $\mathrm{x}$ week $\mathrm{x}$ measurement interaction addressed the question of whether the effects of MSET changed when it was used more often (either increase or decrease). All analyses were carried out using SPSS 10.0.7 (SPSS, Inc., Chicago, IL). An alpha level of 0.05 was used for all statistical tests.

\section{Results}

All participants complete intended intervention and were analysed for the primary outcomes. The means and standard deviations for both the lounge (control) and MSET (experimental) group were calculated for both week 1 and 4 by taking the mean of all 16 values regarding that week, time of reading and variable being tested (Table 1). There was a significant treatment by pre-post interaction found for pulse $(\mathrm{p}=.001)$, despite the small sample sizes (Figure 1). Throughout the four-week period, the nurses assigned to relax in the lounge tended to show either no change in pulse rate or showed an increase. In contrast, the MSET group showed decreases in pulse rate, which became more marked as time went on. During the first week, the mean pre-post reduction in pulse rate in the MSET group was one beat. During the last week, the mean reduction in the MSET group was 10 beats (Table 1). The treatment $\mathrm{x}$ week interaction was also significant $(\mathrm{p}=.017)$. Since the MSET group tended to show higher pulse rates during the pre-measurement, an analysis of covariance was used to control for possible bias. After controlling for the prepulse rate, the groups still showed significantly different pulse rates at post-measurement $(\mathrm{p}=.049)$. Similar differences between groups were found for the psychological measures. Both groups tended to show reductions in anxiety over time from week 1 to week 4 (Table 1). Prepost differences were significantly greater for the MSET group on the State scale $(\mathrm{p}<.001)$ and the Trait scale $(\mathrm{p}=.015)$. The magnitude of the pre post differences is shown in Figure 2.

Table I Means and standard deviations (SD) for the control and experimental groups at weeks I and 4

\begin{tabular}{|c|c|c|c|c|c|c|c|c|}
\hline \multirow{5}{*}{ Pulse reading } & \multicolumn{4}{|c|}{ Control group } & \multicolumn{4}{|c|}{ Experimental group } \\
\hline & \multicolumn{2}{|l|}{ Week one } & \multicolumn{2}{|l|}{ Week four } & \multicolumn{2}{|l|}{ Week one } & \multicolumn{2}{|l|}{ Week four } \\
\hline & $\begin{array}{l}\text { Pre mean } \\
\text { (SD) }\end{array}$ & $\begin{array}{l}\text { Post } \\
\text { mean(SD) }\end{array}$ & $\begin{array}{l}\text { Pre mean } \\
\text { (SD) }\end{array}$ & $\begin{array}{l}\text { Post mean } \\
\text { (SD) }\end{array}$ & $\begin{array}{l}\text { Pre mean } \\
\text { (SD) }\end{array}$ & $\begin{array}{l}\text { Post mean } \\
\text { (SD) }\end{array}$ & $\begin{array}{l}\text { Pre mean } \\
\text { (SD) }\end{array}$ & $\begin{array}{l}\text { Post mean } \\
\text { (SD) }\end{array}$ \\
\hline & 7I & 75 & 69 & 71 & 74 & 73 & 79 & 69 \\
\hline & -11 & -8 & -8 & -6 & -12 & -10 & -9 & -10 \\
\hline \multirow{2}{*}{ State Scale } & 37 & 37 & 38 & 38 & 44 & 28 & 40 & 28 \\
\hline & -9 & -12 & -11 & -11 & -9 & -7 & -7 & -6 \\
\hline \multirow{3}{*}{ Trait Scale } & 43 & 42 & 41 & 39 & 41 & 38 & 36 & 35 \\
\hline & -9 & -7 & -9 & -7 & -6 & -5 & -6 & -4 \\
\hline & 49 & 48 & 38 & 38 & 42 & 33 & 28 & 21 \\
\hline
\end{tabular}

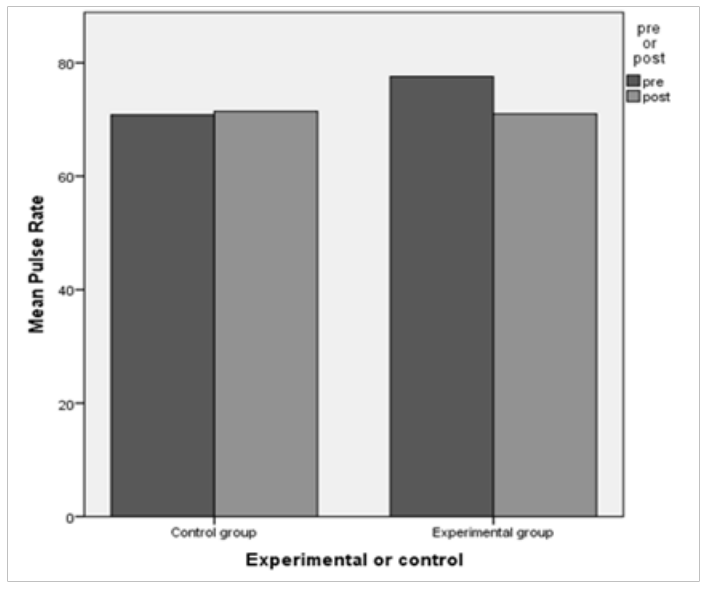

Figure I Comparison of mean pre and post pulse rates.

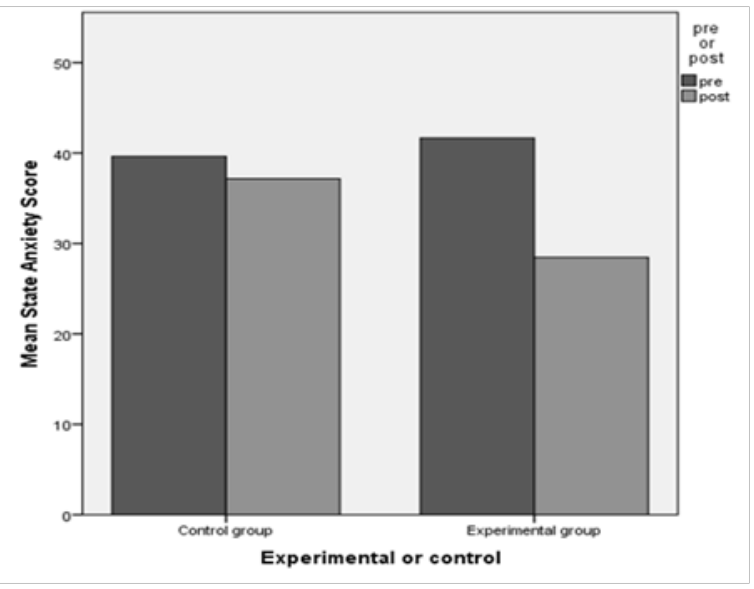

Figure 2 Comparison of mean pre and post state anxiety score. 
The treatment $\mathrm{x}$ week $\mathrm{x}$ measurement interaction was also significant ( $\mathrm{p}=.005)$ which was due to the unit lounge group showing almost no change by week 4 . On the Trait scale and the POMS, the treatment $\mathrm{x}$ measurement interactions were less marked $(\mathrm{p}=.063$ and $\mathrm{p}=.16$, respectively). However, in the case of the POMS, the post scores on the Confusion-Bewilderment sub-scale were consistently lower in the MSET group ( $\mathrm{p}=.004)$. After controlling for confused - bewilderment pre score, the difference between the groups on the post measurement was still significant $(\mathrm{p}=.01)$. Therefore it can be concluded that there is a significant difference between lounge (control) and MSET (experimental) group when all measurements were taken and when pulse and State Anxiety was measured. Cluster analysis was carried out to see if the measurements obtained divided the participants into control and experimental groups. Before the analysis was carried out new variables were created with contained the difference in pre/post scores for each of the 8 sessions. This analysis produced a dendrogram which shows how the participants can be split based on their measurements. To produce this, the within groups cluster method was used with the Euclidean distance.

Figure 3 suggests a dendrogram with two clear clusters, outlined in bold, when calculated only for the State Anxiety and Pulse measurements. Since the numbers 1 and 2 on the y axis denote whether the measurement belongs to the experimental group or control group, it is clear that the clusters split into the two groups reasonably well. From Figure 3 it can be seen that participant number 9 (ID number 415) showed traits of being in the experimental group whilst belonging to the control group within the State anxiety and experimental group whilst belonging to the control group within the State anxiety and Pulse measurement groups meanwhile numbers 7 and 15 (ID numbers 612 and 413) belong to the experimental group but show a control group response. This dendrogram shows further evidence that there are clearly defined groups between control and experimental groups when just looking at the State Anxiety and Pulse measurements. In terms of satisfaction, all of the nurses in the MSET group were unanimously satisfied with the intervention, reporting a reduction in stress on the job and outside of work. Nurses in the MSET group wanted to continue receiving the intervention three times per week or whenever they desired, in contrast to the fixed time intervals of two sessions per week. The majority of nurses who attended the MSET group reported that their job performance was enhanced. In particular, nurses in the MSET group reported that they were more caring toward their patients, that there was improvement in interpersonal relationships and that there was an increase in creative or imaginative on-the-job problem solving.

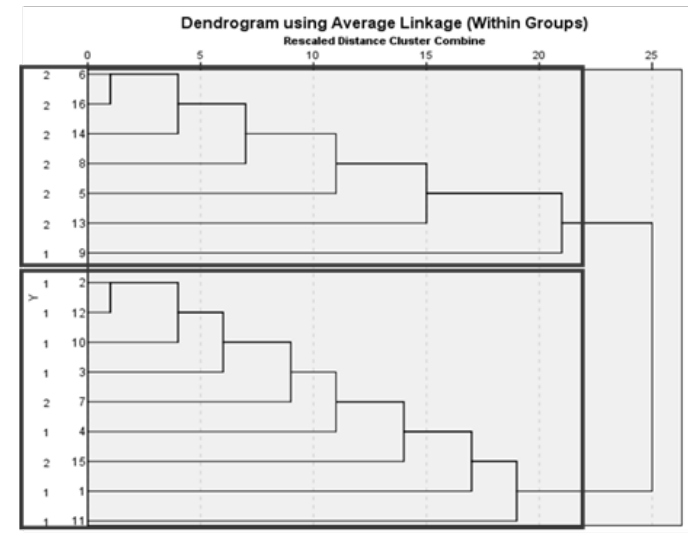

Figure 3 Dendrogram of measurements state anxiety and pulse.

\section{Discussion}

The findings suggest that MSET may be effective as a relaxation therapy for non-clinical populations who experience job stress, such as nurses. Consistent with the hypothesis, nurses in the MSET group showed consistently lower pulse rates after each session, while the unit lounge group showed either no change or an increase. This could support the hypothesis that MSET promotes the occurrence of the relaxation response $\mathrm{e}^{19}$ and corroborates similar reductions in pulse rates from prior MSE research. ${ }^{15}$ It is also noteworthy that pulse rates, a physiological indicator of anxiety, showed greater decreases from pre to post measurement over time for the MSET group. During week one there is a one beat difference between the MSET group and the unit lounge group. By week four there is a 12 beat difference in pulse rates between the two groups indicating that the effects of MSET increase or deepen over time. An explanation of this finding is that respondent conditioning may have occurred, resulting in the evoking of the relaxation response in a stronger manner over time.

The findings suggest that MSET can improve psychological wellbeing by lowering anxiety more than the unit lounge for nurses. Both groups reported a reduction in anxiety. This is related to the effects of stimulus control. Both the unit lounge and the Snoezelen treatment room remove nurses from their stressful work environment and, in turn, negative antecedent and consequential stimuli. These results demonstrate that reducing negative antecedent and consequential stimuli is helpful in reducing anxiety and improving mood for nurses. However, MSET appears to be more effective at reducing anxiety than simply changing one's environment. Differences between groups were less marked using the STAI A-Trait scale and the POMS. This suggests that MSET may be less effective for these outcomes. In the case of the Trait scale, this may have been due to the relatively brief period of the intervention. It is possible that longer or more continued exposure to MSET may be needed to see any improvement in trait anxiety. With regard to the overall POMS score, the problem may lie with its relatively low level of reliability of the instrument. MSET does improve mood in terms of the Confusion-Bewilderment subscale of the POMS. This sub-scale assesses a mixture of cognitive and affective states that are related to "bewilderment and muddleheadedness". ${ }^{30}$ The ability of MSET to reduce these symptoms corroborates researchers who have studied the use of MSEs with elderly dementia patients, concluding that MSET has a cognitive organizing effect. ${ }^{31}$

Qualitatively, nurses expressed high rates of satisfaction with the intervention, felt that the intervention had utility in reducing job stress, and felt the beneficial effects of MSET lasted well beyond the MSE session, with some nurses reporting reduced stress outside of work. Noteworthy is the self-report of the nurses themselves, that by attending MSET they felt more caring toward their patients and reported an increase in creative or imaginative on-the-job problem solving. MSET appears to be very rewarding for nurses to experience. Based on a behavioral paradigm, the effects of reinforcement appear to be operational. That is, nurses in the MSET group are demonstrating the effects of reinforcement, a strengthening of behavior, as shown by their desire to attend MSET more frequently than offered in the study and/or setting up a schedule of MSET attendance that they themselves control. Two classes of nurse behavior appear to benefit from MSET, increased caring and creativity in problem solving. These effects are supported by research on positive emotions which reveal an increase in cognitive and behavioral response availability ${ }^{32}$ and a beneficial effect on clinical problem solving. ${ }^{28}$ 


\section{Conclusion}

The current study provided an examination of the novel use of MSET for nurses to reduce on-the-job stress. As hypothesized the study demonstrates that MSET may be useful in improving clinical and psychological indicators of stress and anxiety in nurses. The results suggest that MSET may reduce stress quickly without the need for special training, unlike biofeedback or yoga. A benefit of intervention is the lack of mediation by other people, support groups, massage, where by Occupational Therapists can facilitate a stress management strategy which, in turn, may increase utilization. This finding may be important for rehabilitation in occupations that are both stressful and solitary in nature. The current results suggest a unique finding, cognitive effects, a reduction in mental and emotional states associated with confusion and bewilderment. Thus, MSET may offer enhanced benefits over other relaxation interventions by positively influencing cognitive and affective aspects of thinking and mood. Nurses reported high levels of satisfaction and expressed the desire to increase and control intervention utilization. This effect may reveal the benefits of sensory reinforcement, which like all classes of reward, strengthen responding. Qualitative information suggests that the clinical benefits of the MSET intervention extend beyond the actual treatment sessions. Nurses reported additional benefits of MSET in relation to improved caring toward their patients, enhancement of interpersonal relationships and an increase in creative or imaginative on-the-job problem solving. The results support the role that positive emotions play in broadening and building adaptive response options across contextual settings and when interacting with people. ${ }^{32}$ This is of significance since nurses are encouraged to provide emotionoriented care to many special needs populations, such as older people with dementia. The findings suggest a possible cost effectiveness strategy, a single MSET room can be used for both patients and the staff that provide people care. By using a single room for clinical care and staff services the cost of a MSE may be offset by providing evidence based practices to improve patient and staff wellbeing. Occupational Therapists are well placed to facilitate this intervention given their role with occupational well-being and sensory overload.

The study, while unique in its topic of inquiry has common methodological limitations. The small number of participants in this initial investigation is a limiting factor of the study's external validity. Future research should replicate theses initial findings with a larger sample of nurses and examine the effects of the intervention on improvements in nurses' helping behaviors such as empathy and on clinical problem solving. The construct of burnout itself may be accessed directly with use of the Maslach Burnout Inventory. ${ }^{33,34}$ This is significant, for untreated on-the-job stress may worsen into burnout, a billable ICD-1-CM (code Z73.0) psychiatric illness. Despite the ability of nurses to record their own pulse more standardized instruments for physiological measures is warranted.

\section{Equipment}

Snoezelen ${ }^{\circledR}$ equipment consisted of 1 colored light spray, 1 sensory play set, lavender essential oil, 1 aroma diffuser, 1 solar projector, 1 gel color wheel, 1 bubble tube, 1 maxi interactive bubble tube, 2 interactive optic galaxy panels, 1 shimmering curtain with colored light spray, 1 mirror ball, 2 mobiles, 1 compact disc player, 2 massagers and seating.

\section{Acknowledgements}

Approval was sought by the Institutional Review Board at Mount
Sinai Beth Israel, New York City and Ethics Committee, Faculty of Health Sciences, University of Southampton.

\section{Funding}

The Hope Foundation.

\section{Conflict of interest}

The Author(s) declare(s) that there is no conflict of interest.

\section{References}

1. Delgado C, Upton D, Ranse K, et al. Nurses resilience and the emotional labour of nursing work: An intergrative review of the empirical literature. Int Nurs Stud. 2017;70:71-88.

2. Dominquez E, Rutledge D. Prevalence of secondary traumatic stress among emergency nurses. J Emerg Nurs. 2009;35(3):199-204.

3. Matrunola P. Is there a relationship between job satisfaction and absenteeism? Journal of Advanced Nursing. 1996;23(4):827-834.

4. Maloney C. Critical incident stress debriefing and pediatric nurses:An approach to support the work environment and mitigate negative consequences. Pediatr Nurs. 2012;38(2):110-113.

5. Alexandrova-Karamanova A, Todorova I, Mongomery A, et al. Burnout and health behaviours in health professionals from seven European countries. Int Arch Occup Environ Health. 2016;89(7):1059-1075.

6. Laschinger H, Finegan J, Wilk P. New graduate burnout:The impact of professional practice environment, workplace civility, and empowerment. Nurs Econ. 2009;27(6):377-383.

7. Field T, Quintino O, Henteleff T, et al. Job stress reduction therapies. Alter Ther Health Med. 1997;3(4):54-56.

8. Huff C. Emotional debriefing. Hospitals \& Health Networks. 2006;80(8):38-42.

9. Van der Hek H, Plomp H. Occupational stress management programmes:a practical overview of published effect studies. Oсcup Med. 1997;47(3):133-141.

10. Hulsegge J, Verheul A. Snoezelen another world. Chesterfield: Rompa, USA; 1986.

11. Holtkamp C. Research at the Bernardus Center. Paper presented at the $3^{\text {rd }}$ Snoezelen World Congress, Toronto, Canada; 1999.

12. Collier L, Mcpherson KM, Ellis-Hill C, et al. Multi-sensory stimulation to improve functional performance in moderate to severe dementia:interim findings from a randomized single blind trial. Am J Alzheimers Dis Other Demen. 2010;25(8):698-703.

13. Lotan M, Gold C, Yalon-Chamovitz S. Reducing challenging behavior through structured therapeutic intervention in the controlled multisensory environment (Snoezelen). International Journal of Disability and Human Development. 2009;8(4):372-392.

14. Schofield P, Payne S. A pilot study into the use of a multisensory environment (Snoezelen) within a palliative day care setting. International Journal of Palliative Nursing. 2003;9(3):124-130.

15. Reddon J, Hoang T, Seghal S, et al. Immediate effects of Snoezelen treatment on adult psychiatric patients and community controls. Current Psychology: A Journal for Diverse Perspectives on Diverse Psychological Issues. 2004;23(3):225-237.

16. Staal J, Sacks A, Matheis R, et al. The effects of Snoezelen (multi-sensory behavior therapy) and psychiatric care on agitation, apathy, and activities of daily living in dementia patients on a short term geriatric psychiatric inpatient unit. Int l Journal of Psychiatry Med. 2007;37(4):357-370.

17. Skinner B. Science and human behavior. New York, USA: The free press; 1953. 
18. Vaughan M and Michael J. Automatic reinforcement:an important but ignored concept. Behaviorism. 1982;10(2):217-228.

19. Benson H, Clipper M. The Relaxation Response. Collins, UK; 1976

20. Favell J, McGimsey J, Schell. Treatment of self-injury by providing alternate sensory activities. Analysis and Intervention in Developmental Disabilities. 1982;2(1):83-104.

21. Glow P, Russell A, Kirby N. Sensory reinforcement using paired stimuli from different modalities. Australian Journal of Psychology. 1971;23(2):133-137.

22. Rincover A, Newsom C, Lovaas I, et al. Some motivational properties of sensory stimulation in psychotic children. Journal of Experimental Child Psychology. 1977;24(2):312-323.

23. Staal J, Pinkney L, Rowan D. Assessment of stimulus preference in Snoezelen therapy for the elderly with dementia. British Journal of Occupational Therapy. 2001;66(12):542-549.

24. DeGrandpre R. A science of meaning:can behaviorism bring meaning to psychological science? Am Psychol. 2000;55(7):721-739.

25. Hunter L. Making time and space:the impact of mindfulness training on nursing and midwifery practice. A critical interpretative synthesis. J Clin Nurs. 2016;25(7-8):918-929.

26. Ludwig D, Kabat-Zinn J. Mindfulness in medicine. JAMA. 2008;300(11):1350-1352.
27. Folkman S. Positive psychological states and coping with severe stress Social Science Medicine. 1997;45(8):1207-1221.

28. Isen A, Rosenzweig A, Young M. The influence of positive affect on clinical problem solving. Med Decis Making. 1991;11(3):221-227.

29. Spielberger C, Gorusch R, Lushene R. The state trait anxiety inventory. Palo Alto, California, USA: Consulting Psychologists Press; 1970.

30. McNair D, Lorr M, Droppleman L. Profile of mood states. San Diego, California, USA: Educational and Industrial Testing Service; 1971.

31. Barker R, Dowling Z, Wareing L, et al. Snoezelen:its long-term and short-term effect on older people with dementia. British Journal of Occupational Therapy. 1997;60(5):213-218.

32. Fredrickson B. The role of positive emotions in positive psychology. Am Psychol. 2001;56(3):218-226.

33. Maslach $\mathrm{C}$, Jackson S. Maslach Burnout Inventory. MBI manual. USA Consulting Psychologists Press Inc; 1981.

34. National Institute for Occupational Safety and Health. Exposure to stress: Occupational hazards in hospitals. Washington, DC:U.S. Department of Health and Human Services Centers for Disease Control and Prevention, National Institute for Occupational Safety and Health, DHHS (NIOSH) Publication; 2008. $136 \mathrm{p}$ 